\title{
Working equation of LR-TD-DFT First-order polarization propagator approximation
}

\author{
Jacob Pedersen $^{1}$ and Kurt Mikkelsen ${ }^{1}$ \\ ${ }^{1}$ University of Copenhagen
}

April 12, 2021

\begin{abstract}
We present the derivation of a new response method termed rst order po- larization propagator approximation. The electronic structure is given by a density functional representation. We provide a detailed derivation of the method along with explicit expressions for the relevant integrals and matrix elements.
\end{abstract}

\section{Hosted file}

workingequations (1).pdf available at https://authorea.com/users/407388/articles/517706working-equation-of-lr-td-dft-first-order-polarization-propagator-approximation 